(C) 1984. The Genetical Society of Great Britain

\title{
THE GENETIC ARCHITECTURE OF THE MALE COURTSHIP SEQUENCE IN DROSOPHILA MELANOGASTER
}

\author{
M. F. COLLINS AND J. K. HEWITT \\ Department of Psychology, University of Birmingham, \\ PO Box 363, Birmingham, B15 2TT, UK
}

Received 20.x.83

\begin{abstract}
SUMMARY
Biometrical genetic analysis of the elements of the male courtship sequence in D. melanogaster using diallel and Cavalli cross-breeding designs, revealed that the genetic architecture of the overall level of courtship was characterised by low additivity, sex-linkage and directional dominance for higher levels of courtship. Similar genetic architectures were found for the latter elements of the courtship sequence, with directional dominance for increased amounts of wing vibration, licking, attempted copulation and copulation itself. There was no evidence of sex-linkage for these characters but there was also evidence of some $i$-type epistatic variation for the wing vibration component of the courtship sequence. The first element of the courtship sequence, orientation, showed a different pattern of inheritance, with again low additive genetic variation but directional dominance for low levels of orientation. These fitness type genetic architectures suggest that the individual elements of the courtship sequence have had a recent history of directional selection favouring a shorter phase of orientation and higher expression of the other courtship elements. This may imply that, though orientation will occur in the initial stages of the courtship process, selection favours males that proceed to the later, more important elements of the courtship sequence fastest.
\end{abstract}

\section{INTRODUCTION}

Courtship behaviour of Drosophila has been studied by many workers since the early work of Sturtevant in 1915. Bastock and Manning (1955) have described the courtship behaviour of Drosophila melanogaster. Typically, the male orientates towards or follows the female in the first phase of the courtship sequence, then the male may extend and vibrate a wing towards the female. This behaviour termed wing vibration is thought to serve as a means of species discrimination and arousal for the female (Burnet, Connolly and Dennis, 1971). The male next extends his proboscis and licks the female genitalia; this behaviour is normally followed by attempted copulation. The final phase of the courtship sequence is copulation itself which lasts for about 20 minutes. As well as these male behaviours the female also performs both acceptance and rejection responses to the male courtship (Connolly and Cook, 1973).

Two basic genetic approaches have been taken to study courtship behaviour in Drosophila. One approach is to dissect the behavioural phenotype into its simplest genetic components; this is the single gene approach or neurogenetic approach (Hall, 1982). The neurogenetic approach uses the tools of molecular and developmental genetics to unravel the biochemical basis that permits both the flexibility and rigidity of 
behaviour. The other approach is through quantitative genetics, which seeks the partitioning of phenotypic variability into genetic and environmental sources, without assuming that one or the other is omnipotent. This approach allows inferences to be made about the evolutionary history of the phenotype under study and stresses the variability of many behavioural phenotypes. Although the two approaches may at first seem to be in competition, they should be regarded as complementary as they were by Fisher (1918) who demonstrated the relationship between quantitative and Mendelian genetics in his classic paper.

Divergence under positive and negative selection for several aspects of male courtship behaviour has demonstrated the heritability of sexual behaviour patterns. Manning $(1961,1963)$ in D. melanogaster, Kessler (1968) in D. pseudoobscura and Manning (1968) in D. simulans have selected successfully for high and low mating speed. Kessler (1969) crossed his selected lines in a Cavalli design and found low narrow sense heritabilities and directional dominance for fast mating speed and high percentage mating. Parsons $(1964,1965,1974)$ and Fulkner $(1966,1972)$ have performed biometrical (diallel) analyses on mating speed using inbred lines of $D$. melanogaster, and have detected the presence of additive genetic variation for mating speed as well as directional dominance for fast mating speed. This genetic architecture implies that fast mating speed is a component of high fitness and of evolutionary significance for Drosophila. MacBean and Parsons $(1966,1967)$ selected for copulation duration and subsequently performed a diallel analysis. The response to selection was slow, which is expected for a trait showing low heritability. Parsons and Kaul (1966) found that temperature affected the mating behaviour of homokaryotypes more than heterokaryotypes, illustrating that additive variation is more responsive to environmental change; this is the only example of genotype $\times$ environment interaction reported for Drosophila courtship behaviour in the literature.

Kraemar and Kessler (1975) have described a biometrical model for the interaction of behavioural characteristics, and their model was illustrated by analysis of mating speed of $D$. pseudoobscura derived from crosses of slow and fast mating strains. Their results revealed a genetic architecture of additive genetic variation and directional dominance for fast mating speed. Dow (1977) performed a Cavalli cross for two lines of D. melanogaster that had been selected for wild-type female receptivity and for high mating success of yellow $(y)$ mutant males. The results showed that for male mating success a simple additive-dominance model adequately described the data, dominance being for higher mating success. The genetic models necessary to explain the female receptivity data were more complex with sex linkage, maternal effects, and epistasis as well as additive and dominance effects. McDonald (1979) found low levels of additive genetic variation for "percentage wing vibration" (PWV); however, there was no evidence of directional dominance and unfortunately her analysis did not permit a test for ambidirectional dominance. Cowling (1980) carried out a detailed diallel analysis of the courtship song, finding that interpulse interval and sine song frequency show a high degree of additive variation and little or no dominance variation. Connolly, Burnet, Kearney and Eastwood (1974) have found strain differences for mating speed, latency to copulation, duration of copulation and for both the proportions of time spent in and the bout lengths of the principal components of the courtship sequence. 
TABLE 1

Genetic architecture of mating behaviour in Drosophila: a review of cross-breeding studies

\begin{tabular}{|c|c|c|c|c|c|c|}
\hline Author & Behaviour & ADD & DOM & EPS & RCP & HERIT \\
\hline Parsons, $1964,65,74$ & Mating speed $10^{\prime 1 \mathrm{D}}$ & + & $t^{\mathrm{h}}$ & NT & + & $60 \%{ }^{n}$ \\
\hline Parsons, $1964,65,74$ & Mating speed $20^{\prime 1 \mathrm{D}}$ & + & $t^{h}$ & NT & + & $51 \%^{n}$ \\
\hline Parsons, $1964,65,74$ & Mating speed $30^{\prime 1 D}$ & + & $+^{\mathrm{h}}$ & NT & + & $34 \%^{n}$ \\
\hline Fulker, 1966, 72 & Mating speed ${ }^{1 \mathrm{D}}$ & + & $t^{h}$ & NT & - & $36 \%$ \\
\hline $\begin{array}{l}\text { MacBean \& Parsons, } \\
1966\end{array}$ & Duration of cop. ${ }^{1 D}$ & + & - & NT & + & $10 \%^{n}$ \\
\hline $\begin{array}{l}\text { Hosgood \& Parsons, } \\
1967\end{array}$ & Mating speed $60^{\prime \prime D}$ & + & $+^{\mathrm{h}}$ & NT & + & NE \\
\hline $\begin{array}{l}\text { Hosgood \& Parsons, } \\
1967\end{array}$ & Duration of cop. ${ }^{1 D}$ & + & - & NT & + & NE \\
\hline Kessler, 1969 & Mating speed ${ }^{2 C}$ & + & $+{ }^{\mathrm{h}}$ & - & - & $17 \%^{n}$ \\
\hline Kessler, 1969 & $\%$ mating $^{2 \mathrm{C}}$ & + & $t^{h}$ & + & - & $25 \%^{n}$ \\
\hline $\begin{array}{l}\text { Kraemar \& Kessler, } \\
1975\end{array}$ & Mating speed ${ }^{2 C}$ & + & $+^{\mathrm{h}}$ & NT & + & $39 \%^{m}$ \\
\hline Dow, 1977 & Mating success $^{1 \mathrm{C}}$ & + & $+^{\mathrm{h}}$ & - & + & NE \\
\hline Dow, 1977 & Female receptivity ${ }^{1 \mathrm{C}}$ & + & $t^{h}$ & + & + & NE \\
\hline McDonald, 1979 & PWV ${ }^{1 C}$ & + & - & - & - & $7 \%$ \\
\hline Cowling, 1980 & $\mathrm{ipi}^{10}$ & + & - & NT & NT & $94 \%^{m}$ \\
\hline Cowling, 1980 & Sine song ${ }^{1 D}$ & + & - & NT & NT & $88 \%^{m}$ \\
\hline
\end{tabular}

Key

+ , significant effect; - , non-significant effect; NT, not tested.

Strain: 1. D. melanogaster; 2. D. pseudoobscura.

Design: $\mathrm{D}$, diallel; $\mathrm{C}$, Cavalli.

Heritabilities: $r$, realised herit; $n$, narrow herit; $m$, my calc. herit; $=(1 / 2 D) /(1 / 2 D+E)$; NE not estimated; $h$, dominance for high performance.

This brief review of biometrical genetic analyses of the genetic architecture of courtship behaviour in Drosophila summarised in table 1 shows in general a low narrow sense heritability and directional dominance for higher mating speed. This type of genetic architecture is associated with phenotypes of high fitness (Broadhurst and Jinks, 1974) and is indicative of a past selective history favouring fast mating speed. The results of Cowling (1980), who found only high additivity for the components of the courtship song, suggest that either the observed variations in courtship song are not critical in terms of fitness or that there is no history of directional selection.

Manning (1967) has described courtship as a process whereby a male provides a female with a stream of stimuli whose effects summate, finally reaching a critical level at which the female accepts copulation. Bastock and Manning (1955) have suggested that neural centres controlling the courtship elements have different thresholds for firing and are representative of the level of physiological excitation of the flies. This process, termed female courtship summation, has recently been criticised by Robertson (1982), who repeated some of the earlier experiments that lead to this theory. He found that short male latencies to courtship lead to longer courtship durations and inferred that the courtship duration was related to the state of agitation of the female. It may be predicted from a theory of female courtship summation that orientation alone without wing vibration, licking or attempted copulation would occur at low excitation, while wing vibration, licking, attempted copulation and copulation itself at successively higher 
levels of excitation. However, very little is known about the heritability of the actual courtship elements as most biometrical genetic analyses have been restricted to the measurement of mating speed and copuation duration. It is therefore of much interest to investigate the genetic architecture of these elements of the courtship sequence, as these elements may reflect the degree of arousal and receptivity of both the male and female. In this paper we report an analysis of the genetic architecture of the individual elements of the male courtship sequence using first the diallel design of Jinks (1954) and subsequently a more detailed Cavalli cross (Cavalli, 1952) between two inbred lines from the diallel study.

\section{MATERials AND METhods}

(i) Details of the strains

Inbred strains of $D$. melanogaster were used from the stocks available in the Department of Genetics at the University of Birmingham. All strains (Texas 1, Texas 15,6C/L, Samarkand and Edinburgh) were maintained by mass mating in standard $1 / 3$ pint milk bottles on standard oatmeal molasses agar medium with some yeast and a strip of paper added to encourage mating and egg laying. Experimental flies were derived from the crossing of 15 three-day-old virgin males with 15 three-day-old virgin females in $1 / 3$ pint milk bottles on standard medium. The parents were removed from the bottles after five days, their progeny were sampled as necessary for five days after the first observation of eclosion. Flies were collected and separated into sexes within the first six hours of eclosion. The sexes were stored separately in groups of 15 in fresh $7.5 \mathrm{~cm} \times 2.5 \mathrm{~cm}$ vials with standard medium and yeast until three days old. All behavioural measurements were recorded in the first five hours of a 12 hour day/night light cycle at $25^{\circ} \mathrm{C} \pm 2{ }^{\circ} \mathrm{C}$.

\section{(ii) Courtship testing procedure}

The continuous recording of single pairs of flies in a biometrical analysis would be impractical given the large numbers of scores required; therefore a time-sampling procedure was developed to score male courtship behaviour. This time-sampling procedure has been validated by Collins, Hewitt and Gogarty (1984); the time-sampled observations of male courtship correlate highly with continuous observations of male courtship. A plastic mating wheel of diameter $10 \mathrm{~cm}$ with ten separate observation chambers (Hotta and Benzer, 1976) each of area $0.64 \mathrm{~cm}^{2}$ and volume $0.38 \mathrm{~cm}^{3}$ was used to observe the courtship behaviour of ten pairs of flies simultaneously.

The flies were first lightly anaesthetised with $\mathrm{CO}_{2}$ to facilitate transfer to the wheel using a soft paint brush. The wheel itself is such that it consists of a plastic disc of ten holes for males and a second disc out of register with ten holes for females, two further outer discs with one hole each allow flies to be placed in either male or female chambers. A wheel is then placed in a wooden stand to prevent movement of the wheel. The wheel and block are placed $30 \mathrm{~cm}$ directly under an anglepoise lamp of $60 \mathrm{~W}$ producing a constant illumination of 300 lux (27.9 foot candles). The flies are allowed to accommodate to the wheel for five minutes. The two inner discs are then 
rotated to bring the male and female chambers into register producing chambers sufficient for courtship behaviour of which observations and recordings are then made. After each test, the wheels are dismantled and then washed in alcohol, in warm water and then in alcohol again to remove any traces of odours.

For all courtship tests the male behaviour was split into four easily identifiable components; orientation, wing vibration, licking and attempted copulation and copulation itself (Bastock \& Manning, 1955). Orientation is maintained throughout the courtship sequence, with the other courtship elements of wing vibration, licking and attempted copulation being superimposed upon it. It follows that the measure of orientation should exclude any orientation which has wing vibration, licking or attempted copulation superimposed upon it, as these reflect higher levels of courtship excitation, although orientation with the other courtship elements superimposed could of course be analysed if desired. It was also decided to score licking and attempted copulation together as one category, as it is known that licking is usually followed by attempted copulation (Burnet and Connolly, 1974) and licking is a behaviour of short duration during the sequence which would not be detected reliably by the time-sampling procedure described below. The courtship behaviour of a pair of flies was noted every 30 seconds. The courtship behaviour was observed over a ten minute period giving each male 20 courtship scores.

A courtship index (CI) is defined for a given male as the fraction of observation periods that showed any courtship behaviour. Four further courtship indexes are defined as follows:

(a) Orientation index (OI), the fraction of observation periods that a given male shows orientation behaviour alone without wing vibration, licking or attempted copulation.

(b) Wing vibration index (WVI), the fraction of observation periods that a given male displays any wing vibration.

(c) Licking and attempted copulation index (LACI), the fraction of observation periods that a given male displays any licking or attempted copulation.

(d) Copulation index (COPI), the fraction of observation periods where a given male is seen to copulate.

These four indexes sum to the courtship index, $\mathrm{CI}=$ $\mathrm{OI}+\mathrm{WVI}+\mathrm{LACI}+\mathrm{COPI}$.

\section{(iii) Diallel design}

To investigate the genetic architecture of time-sampled male courtship in $D$. melanogaster, a $4 \times 4$ diallel analysis was performed. The four inbred lines used were Texas $1(\mathrm{~T}), 6 \mathrm{C} / \mathrm{L}(6)$, Samarkand (S) and Edinburgh (E). A $4 \times 4$ diallel cross consists of crossing all combinations of inbred lines yielding 16 genotypes: four parental genotypes and $12 \mathrm{~F}_{1}$ 's. The experiment was performed in a completely randomised block design, with five courtship pairings observed for each male genotype (in the same observation wheel) in each of seven replicate diallels. Thus a total of $5 \times 16 \times 7=560$ courtship pairings were scored. One critical factor in the observation of male courtship is the choice of the genotype of the female to whom the male should address 
his courtship. The female may be the same genotype as the male, there may be a standard female tester genotype for all male genotypes observed, or the male courtship may be reflected across a panel of female tester genotypes. There are advantages and disadvantages with each procedure and in this study it was decided to use a female of the same genotype as the male. Virgin males were individually isolated for 30 minutes in a mating wheel before transfer to a new wheel containing virgin females. The 30 minute isolation period was made as part of a larger experiment investigating the genetic architecture of male courtship modification by fertilised females (Siegel and Hall, 1979).

\section{(iv) Cavalli design}

A Cavalli cross between Texas 1 and Edinburgh was performed. The experimental design consisted of a 20 blocks of five replicates (half a wheel) of Edinburgh (P1), Texas 1 (P2), $F_{1}$ and $R F_{1}$ (100 pairs of each); 80 blocks of five replicates of the $F_{2}$ ( 400 pairs); and 40 blocks of five replicates each of the backcross generations, $B_{1}, R_{1}, B_{2}$ and $R_{B_{2}}$ (200 pairs of each generation). A total of 1600 pairs of flies were observed in this experiment. The testing procedure for the Cavalli cross is identical to that for the diallel, except for the choice of Texas 15 as a standard female tester genotype. In the diallel analysis the male courtship was tested with females of the same genotype. In the Cavalli design genetic segregation in the $F_{2}$ and backcross generations does not allow an identical female genotype to be used to measure male courtship. It was therefore decided to use a standard female genotype as the tester of male courtship in each generation of the Cavalli design.

Table 2 shows the genetic parameters expected for the Cavalli generation means for a male phenotype; these parameters are all defined in Mather and Jinks (1982). Table 3 shows the genetic and environmental parameters expected for the Cavalli generation variances for a male phenotype and these are again defined in Mather and Jinks (1982). For a male phenotype, sex-linkage may be distinguished from maternal effects only by dissection of the backcross variances. Maternal effects and sex-linkage have the same expectations for generation means. Maternal effects do not contribute to

TABLE 2

Genetic models for Cavalli analysis-means

\begin{tabular}{cl}
\hline Generation & \multicolumn{1}{c}{ Expectations } \\
\hline $\mathrm{P}_{1}$ & $m+[d]+[i]+\left[d_{x}\right]+\left[d_{m}\right]$ \\
$\mathrm{P}_{2}$ & $m-[d]+[i]-\left[d_{x}\right]-\left[d_{m}\right]$ \\
$\mathrm{F}_{1}$ & $m+[h]+[l]+\left[d_{x}\right]+\left[d_{m}\right]$ \\
$\mathrm{RF}_{1}$ & $m+[h]+[l]-\left[d_{x}\right]-\left[d_{m}\right]$ \\
$\mathrm{F}_{2}$ & $m+\frac{1}{2}[h]+\frac{1}{4}[l]$ \\
$\mathbf{B}_{1}$ & $m+\frac{1}{2}[d]+\frac{1}{2}[h]+\frac{1}{4}[i]+\frac{1}{4}[j]+\frac{1}{4}[l]+\left[d_{x}\right]+\left[d_{m}\right]$ \\
$\mathrm{RB}_{1}$ & $m+\frac{1}{2}[d]+\frac{1}{2}[h]+\frac{1}{4}[i]+\frac{1}{4}[j]+\frac{1}{4}[l]$ \\
$\mathbf{B}_{2}$ & $m-\frac{1}{2}[d]+\frac{1}{2}[h]+\frac{1}{4}[i]-\frac{1}{4}[j]+\frac{1}{4}[l]-\left[d_{x}\right]-\left[d_{m}\right]$ \\
$\mathrm{RB}_{2}$ & $m-\frac{1}{2}[d]+\frac{1}{2}[h]+\frac{1}{4}[i]-\frac{1}{4}[j]+\frac{1}{4}[l]$ \\
\hline
\end{tabular}

These parameters are defined in Mather and Jinks (1982) 
TABLE 3

Genetic models for Cavalli analysis_variances

\begin{tabular}{clc}
\hline Generation & \multicolumn{1}{c}{ Expectation } \\
\hline $\mathrm{P}_{1}$ & $E$ & \\
$\mathrm{P}_{2}$ & $E$ & \\
$\mathrm{~F}_{1}$ & $E$ & \\
$\mathrm{RF}_{1}$ & $E$ & \\
$\mathrm{~F}_{2}$ & $E+\frac{1}{2} D+\frac{1}{4} H+\frac{1}{4} I+\frac{1}{16} L+D_{x}$ \\
$\mathrm{~B}_{1}$ & $E+\frac{1}{4} D+\frac{1}{4} H-\frac{1}{2} F+\frac{1}{16} I+\frac{1}{16} L$ \\
$\mathrm{RB}_{1}$ & $E+\frac{1}{4} D+\frac{1}{4} H-\frac{1}{2} F+\frac{1}{16} I+\frac{1}{16} L+D_{x}$ \\
$\mathbf{B}_{2}$ & $E+\frac{1}{4} D+\frac{1}{4} H+\frac{1}{2} F+\frac{1}{16} I+\frac{1}{16} L$ \\
$\mathrm{RB}_{2}$ & $E+\frac{1}{4} D+\frac{1}{4} H+\frac{1}{2} F+\frac{1}{16} I+\frac{1}{16} L+D_{x}$ \\
\hline
\end{tabular}

These genetic parameters are defined in Mather and Jinks (1982)

the genetic variation of the generations in the Cavalli cross. The sex-linkage component contributes only to the variances of the reciprocal backcross generations (these are the crosses of the reciprocal $F_{1}$ back to the parental inbred lines giving the $R_{1}$ and $R B_{2}$ generations). The sex-linkage component does not contribute to the variation of the first backcross generations, which are the $F_{1}$ crosses to the parental lines yielding the $B_{1}$ and $B_{2}$ generations. An appropriate test for sex-linkage is therefore a comparison of the reciprocal backcross and backcross variances. However, a preliminary power analysis suggested that a very large experimental design would be necessary to discriminate between these two effects.

\section{RESUltS}

\section{(i) Diallel analysis}

The means of the diallel cross for each element of the male courtship sequence are given in table 4 and each mean is based on the courtship of 35 pairs of flies. Also shown in table 4 is the number of flies reaching copulation within the ten minute test period. Examining these data first, it is evident that the level of courtship success (defined as the percentage of flies achieving copulation within ten minutes) varies dramatically from 14 per cent in the Texas 1 inbred line to 51 per cent courtship success in the $F_{1}$ cross between Edinburgh and $6 \mathrm{C} / \mathrm{L}$. It is also clear that the $\mathrm{F}_{1}$ crosses considerably outscore their parental inbred lines $\left(P_{i}=20\right.$ per cent; $F_{1}=36$ per cent). This signifies a considerable directional dominance component for male courtship success.

In the Hayman analyses of variance (Hayman, 1954) of these data, shown in table 5, that for courtship success is based on the total number of flies copulating and there is no replication of these data. Therefore the $c$ and $d$ terms of the analysis of variance have been pooled to act as an error term for the main genetic effects. The a term in the analysis of variance tests for additive genetic variation between the lines and the $b$ item is a test for overall dominance. The $b$ item may be further split into three components: $b 1$ is a test for directional dominance, b2 tests for asymmetric gene distributions and the $b 3$ term is made up of any residual dominance variation. The Hayman analysis of variance indicates both additive genetic 
TABLE 4

Means for diallel analysis of male courtship behaviour

\begin{tabular}{cccccccc}
\hline \multicolumn{2}{c}{ Parents } & & & & & & \\
Male & Female & CI & OI & WVI & LACI & COPI & No COP \\
\cline { 1 - 4 } T & T & 0.71 & 0.30 & 0.30 & 0.05 & 0.06 & 5 \\
T & 6 & 0.75 & 0.34 & 0.25 & 0.07 & 0.09 & 10 \\
T & S & 0.68 & 0.31 & 0.24 & 0.06 & 0.07 & 11 \\
T & E & 0.72 & 0.36 & 0.21 & 0.06 & 0.09 & 11 \\
6 & T & 0.75 & 0.30 & 0.29 & 0.08 & 0.08 & 10 \\
6 & 6 & 0.75 & 0.31 & 0.31 & 0.07 & 0.07 & 8 \\
6 & S & 0.75 & 0.30 & 0.29 & 0.07 & 0.09 & 13 \\
6 & E & 0.78 & 0.31 & 0.28 & 0.05 & 0.14 & 16 \\
S & T & 0.79 & 0.34 & 0.32 & 0.08 & 0.05 & 12 \\
S & 6 & 0.79 & 0.35 & 0.29 & 0.08 & 0.07 & 13 \\
S & S & 0.71 & 0.33 & 0.28 & 0.05 & 0.05 & 7 \\
S & E & 0.79 & 0.30 & 0.34 & 0.08 & 0.08 & 12 \\
E & T & 0.86 & 0.31 & 0.41 & 0.07 & 0.07 & 12 \\
E & 6 & 0.82 & 0.32 & 0.34 & 0.05 & 0.13 & 18 \\
E & S & 0.82 & 0.36 & 0.30 & 0.05 & 0.11 & 13 \\
E & E & 0.73 & 0.31 & 0.31 & 0.07 & 0.05 & 8 \\
\hline
\end{tabular}

Each mean is based on 35 pairs of flies

TABLE 5

Hayman analyses of variance for diallel analysis of male courtship

\begin{tabular}{|c|c|c|c|c|c|c|c|c|c|c|}
\hline & & \multicolumn{3}{|c|}{ CI } & \multicolumn{3}{|c|}{ OI } & \multicolumn{3}{|c|}{ WVI } \\
\hline \multicolumn{2}{|c|}{ Source } & MS & $F$ & $\mathbf{P}$ & MS & $F$ & $\mathbf{P}$ & MS & $F$ & $\mathbf{P}$ \\
\hline a & 3 & 0.0179 & $7 \cdot 2$ & *** & 0.0009 & $<1$ & ns & 0.0059 & $2 \cdot 3$ & ns \\
\hline b & 6 & 0.0124 & $5 \cdot 0$ & $* * *$ & 0.0015 & $<1$ & ns & 0.0024 & $1 \cdot 0$ & ns \\
\hline bl & 1 & 0.0525 & $21 \cdot 0$ & $* * *$ & 0.0048 & $2 \cdot 0$ & ns & 0.0001 & $<1$ & ns \\
\hline b2 & 3 & 0.0071 & $2 \cdot 8$ & $*$ & 0.0009 & $<1$ & ns & 0.0045 & $1 \cdot 7$ & ns \\
\hline b3 & 2 & 0.0002 & $<1$ & ns & 0.0008 & $<1$ & ns & 0.0004 & $<1$ & ns \\
\hline c & 3 & 0.0419 & 16.7 & $* * *$ & 0.0038 & $1 \cdot 6$ & ns & 0.0504 & $19 \cdot 4$ & $* * *$ \\
\hline $\mathrm{d}$ & 3 & 0.0044 & $1 \cdot 7$ & ns & 0.0093 & $3 \cdot 8$ & $*$ & 0.0147 & $5 \cdot 6$ & $* *$ \\
\hline \multirow{3}{*}{$\begin{array}{l}\text { Blocks } \\
\text { Error }\end{array}$} & 6 & 0.023 & $<1$ & ns & 0.028 & $1 \cdot 2$ & ns & 0.0024 & $<1$ & ns \\
\hline & 90 & 0.0025 & & & 0.0024 & & & 0.0026 & & \\
\hline & & \multicolumn{3}{|c|}{ LACI } & \multicolumn{3}{|c|}{ COPI } & \multicolumn{3}{|c|}{ No COP } \\
\hline \multicolumn{2}{|c|}{ Source } & MS & $\mathbf{F}$ & $\mathbf{P}$ & MS & $F$ & $\mathbf{P}$ & MS & F & $\mathbf{P}$ \\
\hline $\mathbf{a}$ & 3 & 0.0005 & $1 \cdot 0$ & ns & 0.0084 & $2 \cdot 5$ & ns & $12 \cdot 4583$ & $21 \cdot 4$ & $* *$ \\
\hline b & 6 & 0.0013 & $2 \cdot 6$ & $*$ & 0.0076 & $2 \cdot 2$ & $*$ & $19 \cdot 9271$ & $34 \cdot 2$ & $* * *$ \\
\hline b) & 1 & 0.0005 & $1 \cdot 0$ & ns & 0.0228 & 6.7 & $*$ & $93 \cdot 5208$ & 160 & $* * *$ \\
\hline b2 & 3 & 0.0021 & $4 \cdot 2$ & $*$ & 0.0057 & $1 \cdot 7$ & ns & $2 \cdot 4583$ & $4 \cdot 2$ & ns \\
\hline b3 & 2 & 0.0005 & $1 \cdot 0$ & ns & 0.0027 & $<1$ & ns & 9.3333 & 15.9 & $* * *$ \\
\hline c & 3 & 0.0017 & $3 \cdot 4$ & $*$ & 0.0030 & $<1$ & ns & 1.0000 & & \\
\hline d & 3 & 0.0001 & $<1$ & ns & 0.0005 & $<1$ & ns & $0 \cdot 1667$ & & \\
\hline Blocks & 6 & 0.0010 & $2 \cdot 0$ & ns & 0.0031 & $<1$ & ns & $0 \cdot 583^{a}$ & & \\
\hline Error & 90 & 0.0005 & & & 0.0035 & & & & & \\
\hline
\end{tabular}

${ }^{a}$ pooled (c \& d) error term for d d.f. 
variation $(F(3,90)=21 \cdot 4, P<0.01)$ and dominance variation $(F(6,90)=$ $34.2 \mathrm{P}<0.001)$ for the number of pairs of flies achieving copulation. The dominance variation is primarily composed of a strong directional dominance component $(F(1,90)=160, P<0.001)$ with some residual dominance variation $(F(2,90)=15.9, P<0.01)$. The direction of dominance is for increased mating success. This is the type of genetic architecture previously found by diallel analysis of mating speed (Parsons, 1964; Fulker, 1966; Kraemar and Kessler, 1975) and suggests that selection favours high expression of mating ability and obviously implies that mating ability is very closely related to fitness.

The results of the diallel analysis of the components of the courtship sequence are also found in table 4 (the means) and table 5 (the Hayman analyses of variance). For $\mathrm{CI}$, the overall courtship index, the Hayman analysis reveals some reciprocal effects (c item, $\mathrm{F}(3,90)=16 \cdot 7, \mathrm{P}<0.001$ ) which may be ascribable to either maternal effects or to sex-linkage. A brief glance at the means for CI reveals that these reciprocal effects are mainly due to two inbred lines, Texas 1 and Edinburgh. There is evidence for some additive genetic effects $(\mathrm{F}(3,90)=7 \cdot 2, \mathrm{P}<0.001)$ and also dominance variation $(F(6,90)=5 \cdot 0, P<0.001)$. The dominance variation is again primarily composed of a strong directional dominance component $(F(1,90)=21 \cdot 0$, $\mathrm{P}<0.001)$ but there is also some evidence for an asymmetrical gene distribution $(\mathrm{F}(3,90)=2 \cdot 8, \mathrm{P}<0.05)$ (i.e., an unequal representation of increasing and decreasing alleles in the inbred lines crossed). This genetic architecture with low additivity and directional dominance for increased levels of courtship is similar to that found earlier for courtship success in this study.

Analysis of the orientation index (OI) revealed only variation for the $d$ term in the analysis of variance $(\mathrm{F}(3,90)=3.8, \mathrm{P}<0.05)$. The wing vibration component (WVI) of the courtship display again only revealed significant reciprocal effects (c item, $F(3,90)=19 \cdot 4, P<0.001$; d item, $F(3,90)=5.6$, $P<0.01)$. The combined measure of licking and attempted copulation (LACI) shows a genetic architecture consisting of reciprocal effects (c item, $\mathrm{F}(3,90)=3 \cdot 4, \mathrm{P}<0.05)$ and some dominance variation $(\mathrm{F}(6,90)=2 \cdot 6, \mathrm{P}<$ $0.05)$. The copulation index (COPI) shows only dominance variation $(\mathrm{F}(6,90)=2 \cdot 2, \mathrm{P}<0.05)$; this variation is ascribable to the directional dominance component $\mathrm{bl}(\mathrm{F}(1,90)=6 \cdot 7, \mathrm{P}<0.05)$ and dominance is in the direction of increased levels of copulation. The components of the courtship sequence may be divided into two groups in terms of the results of the diallel analysis, one group (CI, LACI, COPI and number copulating) reveal a pattern of inheritance which is characteristic of a fitness related phenotype whilst the measures of orientation and wing vibration reveal little or no apparent genetic variation. The absence of significant additive or dominance effects for OI and WVI may be due to lack of power in the experimental design or possibly due to a more complex pattern of inheritance involving maternal or sex-linkage effects in the lines studied. These differences in genetic architecture may indicate separate genetic control for these elements of the courtship sequence.

It should be noted that the standard covariance/variance $\left(W_{r} / V_{r}\right)$ graphical interpretation (Jinks, 1954) of the diallel results which tests the suitability of a purely additive/dominance model was not carried out. This was because of the detection of reciprocal effects in the Hayman analyses of variance and also because of the relatively small number of lines used in 
the diallel design. There is therefore no test for epistasis and because of the absence of this test and the presence of reciprocal effects it was decided not to estimate any genetic parameters from the Hayman analysis. Instead the Cavalli cross between two of the inbred lines used in the diallel cross, Edinburgh (P1) and Texas 1 (P2), was performed to investigate in more detail the genetic architecture of male courtship and to provide a possible test between sex-linkage and maternal effects as the source of reciprocal effects found in the diallel analysis. The design would also permit a test for epistatic genetic variation and the estimation of the genetic and environmental sources of variation.

\section{(ii) Cavalli analysis}

The means and variances for each generation of the Cavalli cross for each component in the courtship sequence are shown in table 6. Model fitting to both means and variances for each component of the courtship sequence is shown in table 7. Models were chosen which satisfied the criteria stated by Gale, Mather and Jinks (1977); these were, that the test of goodness of fit of the observed and estimated statistics is non-significant, that all individual components of the model are significant, and, that the magnitudes and signs of the components of the models are statistically and genetically sensible. It is well known that model fitting to second degree statistics (variances) is less reliable than fitting models to first degree statistics (means) (Mather and Jinks, 1982). In some cases, for model fitting to variances, models with $\mathrm{E}$ and $\mathrm{H}$ actually were a better fit than models with $\mathrm{E}$ and $\mathrm{D}$; however, a model of $\mathrm{E}$ and $\mathrm{H}$ alone does not make much genetic sense.

Looking first at the means for the Cavalli data, it is apparent that the $F_{1}$ crosses outscore their parental lines for each component of the courtship sequence. The genetic architecture in the means for $\mathrm{CI}$ reveals additivity and strong directional dominance with sex-linkage or a maternal effect. The best model for the variances included additive effects and sex-linkage, and the presence of sex-linkage is confirmed by direct comparison of the appropriate pooled backcross variances, combined following Winer (1971, p. 377), giving $\left(R B_{1}+R B_{2}\right) /\left(B_{1}+B_{2}\right), F^{\prime \prime}(396,395)=1 \cdot 21, P<0.05$. OI shows a similar genetic architecture to that described for $\mathrm{CI}$, with a low level of additivity, directional dominance and sex-linkage, except that the directional dominance is for decreased levels of orientation only behaviour. This is also reflected in the finding of a negative [d] value indicating that the high scoring parent for overall courtship, Edinburgh, performs less orientation than the less successful courter Texas 1. This may show that the more successful courters, the $F_{1}$ 's, exhibit more overall courtship but actually less orientation and therefore spend more of their time in the later elements of the courtship sequence. Inspection of model fitting to the variances finds significant additive genetic variation and sex-linkage; the comparison of backcross variances testing for sex-linkage confirm the model fitting findings, $F^{\prime \prime}(395,393)=1 \cdot 30, P<0.01$.

The genetic architecture of wing vibration (WVI) shows no sex linkage, but there is directional dominance and some additive genetic variation displayed in the means for high wing vibration. There is also evidence of i-type epistasis which is negative in sign as this would be expected to support heterosis for wing vibration. The best fitting model for the variances reveals 


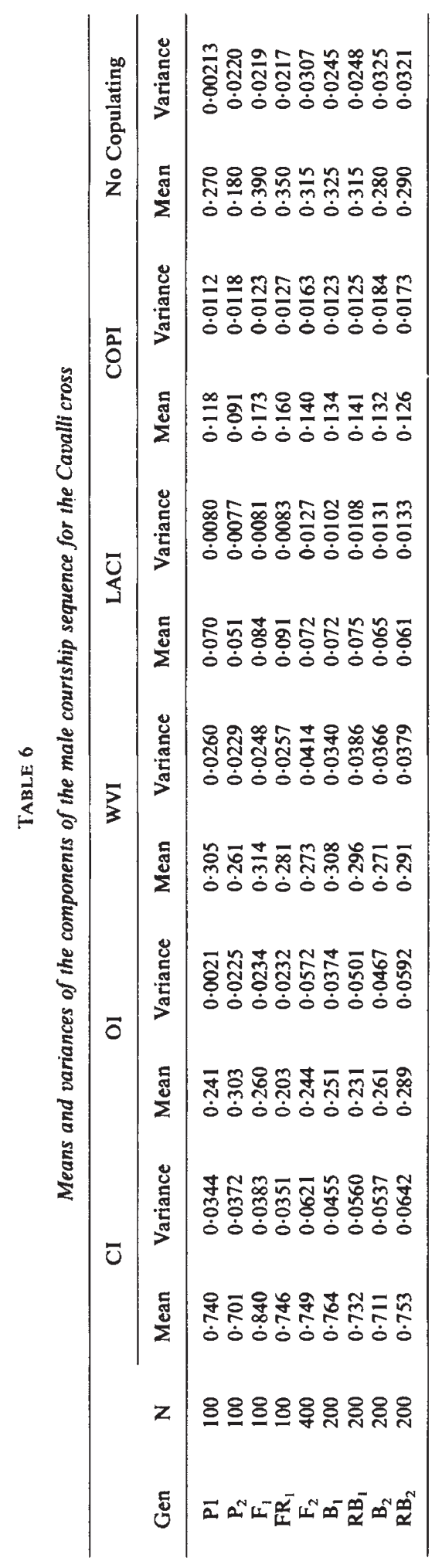




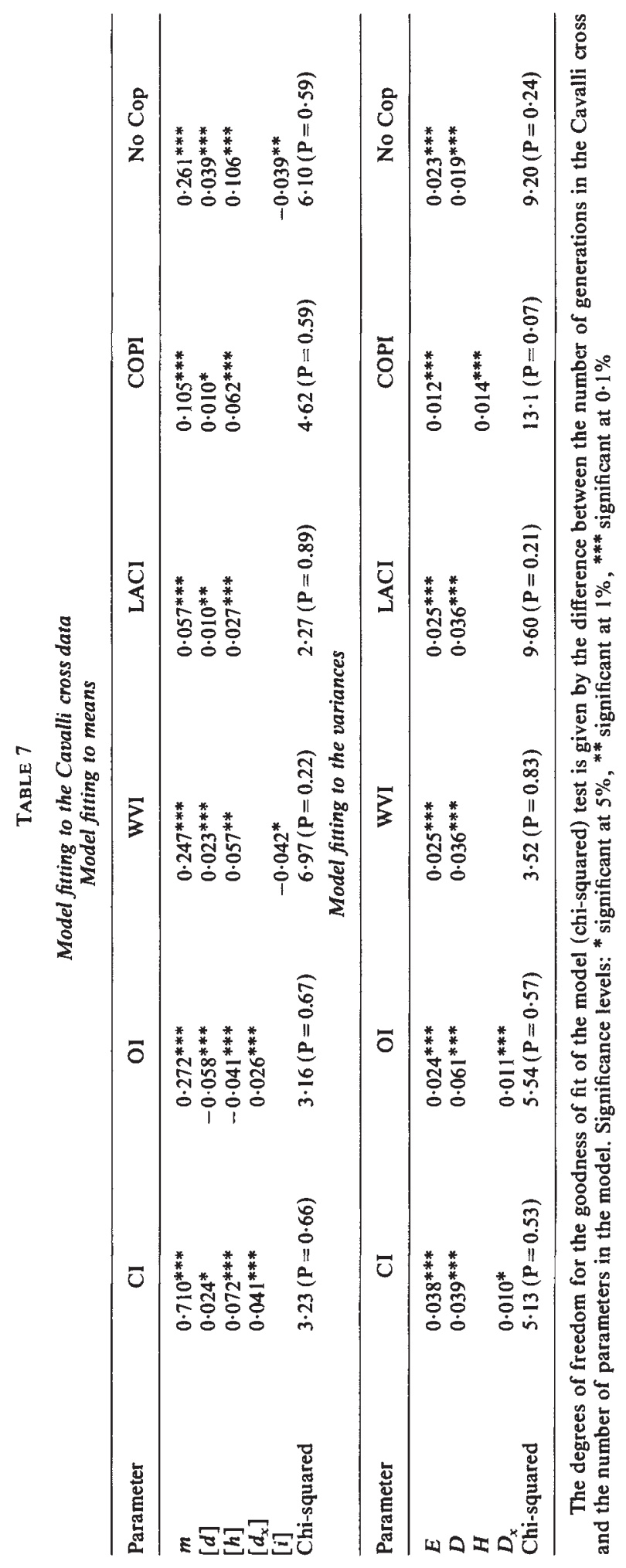


only additive and environmental effects for WVI. For LACI, there is evidence of additivity and directional dominance for increased $\mathrm{LACI}$; the variances show only additivity and environmental sources of variation present. Data on COPI shows low additivity and very strong directional dominance for increased copulation; the only variance model that fits is one with dominance and environmental variation, and the additive variation may be very small or may be masked by linkage disequilibrium (linkage in repulsion would cause an inflation of $\mathrm{H}$ and defiation of $\mathrm{D}$ ). Tests for linkage disequilibrium are complicated and require the comparison of the estimates of $D$ and $H$ over ranks (Mather and Jinks, 1982). Analysis of the number of pairs actually copulating in each generation reveals a conventional fitness type genetic architecture in the first statistics, low additivity, strong directional dominance for high copulation and negative i-type epistasis which causes depression of parental means and further aids any heterotic effect.

Overall, the results of the Cavalli analysis suggest that rapidly initiated and sustained courtship in male Drosophila melanogaster moving quickly to the advanced stages is likely to be highly correlated with reproductive success, with a genetic architecture characterised by low additive genetic variation and strong directional dominance. However, the components of the courtship sequence do not all show the same pattern of inheritance; evidence is found for sex-linkage in this cross for $\mathrm{CI}$ and $\mathrm{OI}$ and there is directional dominance for low OI, whereas for the other courtship elements the dominance is in the high expression.

\section{Discussion}

The results of the diallel analysis show that there is genetic variation for the various components of male courtship in $D$. melanogaster. This genetic variation can be ascribed to three main sources: additive genetic effects, dominance effects and reciprocal effects. The dominance is mainly directional in nature and is for increased and more successful levels of male courtship, although there was no statistically significant evidence of additive or dominance variation for the orientation and wing vibration components of the courtship sequence considered individually; this may be due to lack of power in detecting any genetic variation.

The results of the Cavalli cross resolve some of the questions that the diallel results posed. The increased power of the experimental design has revealed results similar to those found in the diallel cross and has also uncovered genetic variation for the orientation and wing vibration elements of the courtship sequence. It has been possible to ascribe the source of the reciprocal variation found in the diallel analysis to sex-linkage rather than to maternal effects. These sex-linked effects are primarily found in the orientation index but there is also evidence for sex-linkage in the overall courtship measure as well. The analysis has also revealed i-type epistatic variation for WVI and the percentage of flies achieving copulation. This is negative in nature in both cases and contributes to the heterotic effect produced with the strong directional dominance.

The finding of directional dominance for low levels of orientation may illustrate two interesting points. Firstly, that the pattern of inheritance for OI is different from the other elements of the courtship sequence may be used as evidence that the elements of the courtship sequence are under 
independent genetic control. Secondly, the result supports the model of courtship summation proposed by Manning (1967). One indication of inferior male courtship would be higher thresholds for the later elements of the courtship sequence and female acceptance; this would cause higher levels of orientation in less successful males. One may infer that orientation is only important in the initial stages of the courtship process and males which orientate for too long do not perform enough of the more important later elements of the courtship sequence. Manning (1961) found that in his line selected for slow mating speed the level of general activity was high compared with a control line; if the initial orientation behaviour is related to general activity, a result of this type would be expected. Tompkins, Gross, Hall, Gailey and Siegel (1982) have shown that females stop moving to encourage male courtship and copulation once their threshold for male acceptance has been reached. Thus males who court successfully would not orientate for as long as males who were less successful courters.

The results show that the marked depression found in the inbred lines is removed by intercrossing the lines. It would be predicted that selection on a heterogeneous foundation population would produce an asymmetric response, that is selection would make most advances in the direction of poor courtship and there should be little or no response to selection for efficient courtship. In fact, Manning $(1961,1963)$ selected for both fast and slow mating speed in $D$. melanogaster. He found little or no response for fast mating speed but there was a considerable response to selection for slow mating speed, a character which could be mediated by slow initiation of courtship followed by prolonged exhibition of the early elements of the courtship sequence. This asymmetrical response to selection has also been found for behavioural characters in $D$. melanogaster which may be related to fitness, for example, performance in a discriminative odour avoidance task (Hewitt, Fulker and Hewitt, 1983).

These results imply that the ability to display appropriate and vigorous courtship has been closely associated with fitness in the evolution of the $D$. melanogaster genome. If this interpretation is correct, there should be a very low probability of finding new mutations which resulted in increased and more successful courtship. In fact, of the single gene mutants isolated on the grounds of abnormal courtship, all show deficient and reduced courtship performance (Hall, 1981). Cacophony (cac) mutant males produce abnormal courtship song and are blocked at this stage of the courtship sequence. Celibate (cel) and fruitless (fru) males perform generally poor courtship and rarely reach the later stages of the courtship sequence. Two other mutants experience problems in actual copulation; coitus-interruptus (coi) males copulate for only 60 per cent of the normal copulation duration and stuck (sk) males have difficulty in dismounting after copulation.

The level of copulation success of about 20 per cent found in the four inbred lines studied is considerably lower than that found by Connolly, Burnet, Kearney and Eastwood (1974) in their survey of the courtship of three inbred lines. The low level of successful copulations found in this study may be due to the size of the courtship arena, temperature effects, due to the storage of flies in groups prior to testing or may be characteristic of the inbred line studied (Collins, Hewitt and Gogarty, 1984). It is clear however that the courtship phenotype is sensitive to fluctuations in environmental variation. 
Since natural selection acts on the individual as a whole and not necessarily on one particular component of fitness, it would be reasonable to suppose that there may be correlated responses to selection between components of fitness. A high fitness for one character is therefore likely to be associated with high fitnesses for other characters. On this argument a genotype for high mating ability would be expected to be of high fitness for other fitness related characteristics. One possible component of fitness, learning ability (Hewitt, Fulker and Hewitt, 1983) has been directly related to the courtship process by several authors (Pruzan, Applewhite and Bucci, 1977; Siegel and Hall, 1979). In fact a diallel analysis of the modification of courtship by prior experience with a fertilised female revealed a fitness type genetic architecture of low additivity and directional dominance for increased learning ability (Collins and Hewitt, 1981). Experiments are in progress to investigate the relationships between male courtship, male courtship modification and odour discrimination avoidance conditioning.

The interpretation of the observed genetic architectures found for the courtship behaviours are complicated by the nature of the courtship phenotype itself. A courtship phenotype is a composite of both male and female phenotypes and their interaction. The specific roles and interactions between males and females is little understood. We have recently (Collins and Hewitt, 1984) studied in detail the interaction between males and females of four different genotypes using a competition diallel design. The results indicated that a model based on simple additive effects for the male and female genotypes did not explain the pattern of courtship observed across genotypes. There was greater variation between male genotypes than between female genotypes for the courtship elements. Further analysis showed that courtship behaviour was significantly higher between like genotype pairings than between unlike genotype pairings for each courtship element except for orientation where unlike genotype combinations performed more orientation than unlike genotype combinations. There was also evidence for a negative correlation between male and female courtship behaviour within genotypes. Work is presently extending this competition diallel design to allow the dissection of the phenotypic variation into its genetic and environmental components by superimposing a diallel cross on the competition diallel design. This method should prove useful in investigating the genetic architecture of social behaviour in Drosophila as well as other species.

With the possibility of obtaining accurate and rapid information on male courtship behaviour using a time-sampling technique (Collins, Hewitt and Gogarty, 1984), it is now possible to investigate the genetic architecture of the individual elements of the male courtship sequence. These individual elements are shown to be under different genetic control. It should now be possible to use this model of male courtship behaviour to investigate in greater depth the role of genotype-environment interaction in the evolution of behavioural characters closely related to fitness.

Acknowledgements. This work was supported by a SERC research postgraduate studentship to MFC and continued support by a SERC postdoctoral fellowship is alo acknowledged. Statistical analysis was carried out on the ICL 1906A computer at the University of Birmingham. 


\section{REFERENCES}

BASTOCK, M. AND MANNING, A. 1955. The courtship of Drosophila melanogaster. Behaviour, $8,85-111$.

BROADHURST, P. L. AND JINKS, J. L. 1974. What the genetical architecture can tell us about the natural selection of behavioural traits. In J. H. F. van Abeelen (ed.), The Genetics of Behaviour, Amsterdam, North Holland.

BURNET, B. AND CONNOLLY, K. 1974. Activity and sexual behaviour in Drosophila melanogaster. In J. H. F. van Abeelen (ed.), The Genetics of Behaviour, Amsterdam, North Holland.

BURNET, B., CONNOLLY, K. AND DENNIS, L. 1971. The function and processing of auditory information in the courtship behaviour of Drosophila melanogaster. Animal Behaviour, 19, $409-415$.

CAVALLI, L. L. 1952. An analysis of quantitative inheritance. In E. C. R. Reeve and C. H. Waddington (eds.), Quantitative Inheritance, London, HMSO.

COLLINS, M. F. AND HEWITT, J. K. 1981. A biometrical genetical analysis of a reported conditioned response in the courtship behaviour of male Drosophila melanogaster. Behaviour Genetics, 11, 595-596 (Abstr.).

COLliNS, M. F. AND HEWITT, J. K. 1984. Competition diallel analysis of the male courtship sequence in Drosophila melanogaster. Submitted to Evolution

COLLINS, M. F., HEWITT, J. K. AND GOGARTY, J. F. 1984. Validating time-sampled observations of courtship in Drosophila melanogaster for behavior genetic analysis. Behavior Genetics (in press).

CONNOLLY, K. AND COOK, R. 1973. Rejection responses by female Drosophila melanogaster: their ontogeny, causality and effects upon the behaviour of the courting male. Behaviour, $64,142-166$.

CONNOLLY, K., BURNET, B., KEARNEY, M. AND EASTWOOD, L. 1974. Mating speed and courtship behaviour of inbred strains of Drosophila melanogaster. Behaviour, 68, 61-74.

COWLING, D. E. 1980. The genetics of Drosophila melanogaster courtship song-diallel analysis. Heredity, 45, $401-403$.

DOW, M. A. 1977. Selection for mating success of yellow mutant Drosophila melanogaster: Biometrical genetic analysis. Heredity, 38, 161-168.

FISHER, R. A. 1918. The correlations between relatives on the supposition of Mendelian inheritance. Transactions of the Royal Society of Edinburgh, 52, 399-433.

FULKER, D. W. 1966. Mating speed in male Drosophila melanogaster. A psychogenetic analysis. Science, 153, 203-205.

FULKER, D. W. 1972. Applications of a simplified triple test-cross. Behavior Genetics, 2, 185-198. GALE, J. G., MATHER, K. AND JINKS, J. L. 1977. Joint scaling tests. Heredity, 38, 47-51.

HALL, J. C. 1981. Sex behavior mutants in Drosophila. Bioscience, 31, 125-130.

HALL, J. C. 1982. Genetics of the nervous system in Drosophila. Q. Rev. of Biophysics, 15, 223-479.

HAYMAN, B. I. 1954. The analysis of variance of diallel tables. Biometrics, 10, 235-244.

HEWITT, J. K., FULKER, D. W. AND HEWITT, C. A. 1983. Genetic architecture of olfactory discriminative avoidance conditioning in Drosophila melanogaster. Journal of Comparative Psychology, 97, 52-58.

HOTTA, Y. AND BENZER, S. 1976. Courtship in Drosophila mosaics: sex specific foci for sequential action patterns. Proceedings of the National Academy of Sciences of the United States of America, 73, 4154-4158.

JJNKS, J. L. 1954. The analysis of continuous variation in a diallel cross of Nicotiana rustica. Genetics, 39, 767-788.

JINKS, J. L. 1983. Biometrical genetics of heterosis. In R. Frankel (Ed.) Monographs on Theoretical and Applied Genetics, vol 6, Heterosis, Springer-Verlag, Berlin Heidelberg.

KESSLER, S. 1968. The genetics of Drosophila mating behaviour. I. Organisation of mating speed in Drosophila pseudoobscura. Animal Behaviour, 16, 485-491.

KESSLER, S. 1969. The genetics of Drosophila mating behavior. II. The genetic architecture of mating speed in Drosophila pseudoobscura. Genetics, 62, 421-433.

KRAEMAR, H. C. AND KESSLER, S. 1975. A biometrical analysis of a mating characteristic in Drosophila. Heredity, 34, 1-10.

MACBEAN, I. T. AND PARSONS, P. A. 1966. The genotypic control of the duration of copulation in Drosophila melanogaster. Experientia, 22, 101-102.

MACBEAN, I. T. AND PARSONS, P. A. 1967. Directional selection for duration of copulation in Drosophila melanogaster. Genetics, 56, 233-239.

MANNING, A. 1961. The effects of artificial selection for mating speed in Drosophila melanogaster. Animal Behaviour, 9, 82-92. 
MANNING, A. 1963. Selection for mating speed in Drosophila melanogaster based on one sex. Animal Behaviour, 11, 116-120.

MANNING, A. 1967. The control of sexual receptivity in female Drosophila. Animal Behaviour, $15,239-250$.

MANNING, A. 1968. The effects of artificial selection for slow mating in Drosophila simulans. I. The behavioural changes. Animal Behaviour, 16, 108-113.

MATHER, K. AND JINKS, J. L. 1982. Biometrical Genetics 3rd. ed., Chapman and Hall, London. MCDONALD, J. 1979. Genetic analysis of lines selected for wing vibration in Drosophila melanogaster. Behavior Genetics, 9, 579-584.

PARSONS, P. A. 1964. A diallel cross for mating speed in Drosophila melanogaster. Genetica, $35,141-151$.

PARSONS, P. A. 1965. The determination of mating speeds in Drosophila melanogaster for various combinations of inbred lines. Experientia, 21, 478.

PARSONS, P. A. 1974. Male mating speed as a component of fitness in Drosophila. Behavior Genetics, 4, 395-404.

PARSONS, P. A. AND KAUL, D. 1966. Mating speed and duration of copulation in Drosophila pseudoobscura. Heredity, 21, 219-225.

PRUZAN, A., APPLEWHITE, P. B. AND BUCCI, M. J. 1977. Protein synthesis inhibition alters Drosophila mating behavior. Pharmacology, Biochemistry and Behavior, 6, 355-357.

ROBERTSON, H. M. 1982. Female courtship summation in Drosophila melanogaster. Animal Behaviour, 30, 1105-1117.

SIEGEL, R. W. AND HALL, J, C. 1979. Conditioned responses in the courtship behavior of normal and mutant Drosophila. Proceedings of the National Academy of Sciences of the United States of America, 76, 3430-3434.

STURTEVANT, E. H. 1915. Experiments of sex recognition and the problem of sexual selection in Drosophila. Journal of Animal Behaviour, 5, 351-366.

TOMPKINS, L., GROSS, A. C., HALL, J. C., GAILEY, D. A. AND SIEGEL, R. w. 1982. The role of female movement in the sexual behavior of Drosophila melanogaster. Behavior Genetics, $12,295-307$

WINER, B. J. 1971. Statistical principles in experimental design, 2nd. ed., McGraw-Hill, Tokyo. 